\title{
Somatic MED12 mutations are associated with poor prognosis markers in chronic lymphocytic leukemia
}

\author{
Kati Kämpjärvi ${ }^{1, *}$, Tiina M. Järvinen ${ }^{2, *}$, Tuomas Heikkinen ${ }^{1}$, Amy S. Ruppert ${ }^{3}$, \\ Leigha Senter ${ }^{2}$, Kevin W. Hoag ${ }^{2}$, Olli Dufva ${ }^{1}$, Mika Kontro ${ }^{4}$, Laura Rassenti ${ }^{5}$, \\ Erin Hertlein ${ }^{3}$, Thomas J. Kipps ${ }^{5}$, Kimmo Porkka ${ }^{4}$, John C. Byrd ${ }^{3}$, Albert de la \\ Chapelle ${ }^{2}$, Pia Vahteristo ${ }^{1}$ \\ ${ }^{1}$ Department of Medical Genetics and Genome-Scale Biology Research Program, University of Helsinki, Helsinki, Finland \\ ${ }^{2}$ Department of Molecular Virology, Immunology and Medical Genetics, Division of Human Cancer Genetics, Comprehensive \\ Cancer Center at The Ohio State University, Columbus, OH, USA \\ ${ }^{3}$ Department of Internal Medicine, Division of Hematology, Comprehensive Cancer Center at The Ohio State University, \\ Columbus, $\mathrm{OH}$, USA \\ ${ }^{4}$ Hematology Research Unit Helsinki, Department of Medicine, Helsinki University Central Hospital and University of Helsinki, \\ Helsinki, Finland \\ ${ }^{5}$ Moores University of California-San Diego Cancer Center, University of California San Diego, CA, USA \\ *These authors have contributed equally to this work
}

Correspondence to:

Pia Vahteristo, e-mail: pia.vahteristo@helsinki.fi

Keywords: Chronic lymphocytic leukemia, MED12, somatic mutation, cancer genetics, prognosis

Received: October 01,2014 Accepted: November 15, $2014 \quad$ Published: December 18, 2014

\section{ABSTRACT}

Chronic lymphocytic leukemia (CLL) is the most common leukemia in adults. We performed systematic database search and identified highly specific MED12 mutations in CLL patients. To study this further, we collected three independent sample series comprising over $700 \mathrm{CLL}$ samples and screened MED12 exons 1 and 2 by direct sequencing. Mutations were identified at significant frequency in all three series with a combined mutation frequency of 5.2\% (37/709). Positive mutation status was found to be associated with unmutated IGHV and ZAP70 expression, which are well-known poor prognosis markers in CLL. Our results recognize CLL as the first extrauterine cancer type where 5'terminus of MED12 is mutated at significant frequency. Functional analyses have shown that these mutations lead to dissociation of Cyclin C-CDK8/19 from the core Mediator and to the loss of Mediator-associated CDK kinase activity. Additional studies on the role of MED12 mutation status as a putative prognostic factor as well as mutations' exact tumorigenic mechanism in CLL are warranted.

\section{INTRODUCTION}

Chronic lymphocytic leukemia (CLL) is the most prevalent adult leukemia in Western societies. The disease is characterized by the accumulation of monoclonal CD5-, CD19-, and CD23-positive B cells in the blood and lymphoid tissues. The clinical course of patients with this disease is heterogeneous, ranging from highly indolent, which may never require therapy, to relatively aggressive, which may require therapy soon after diagnosis. CLL can be divided into two subclasses based on the occurrence of somatic hypermutation in the immunoglobulin heavy-chain variable (IGHV) genes. [1] The subclass with mutated IGHV genes (M-CLL) has an improved treatment-free and overall survival compared to the subclass with unmutated IGHV genes (U-CLL) [2, 3]. Currently, there is no curative drug therapy for CLL, but chemo-immunotherapy can significantly improve survival [4]. As the course of the disease is highly unpredictable, there is an urgent need to better elucidate the biology of CLL.

Recent large-scale sequencing studies have shown that the average number of somatic mutations in CLL is smaller than in solid tumors but comparable with other hematological neoplasms [5]. Only a few genes (e.g. TP53, NOTCH1, SF3B1, XPO1, BIRC3, MYD88) have 
emerged as frequently mutated in CLL. In contrast, many other genes are recurrently mutated at lower frequencies (2-5\%). There are also some CLL cases which do not have mutations in any known CLL driver gene. [Reviewed in 6] These observations emphasize the molecular heterogeneity of the disease and highlight the need for a comprehensive classification of CLL based on molecular characteristics.

Mediator is a multisubunit protein complex required for activator-dependent transcription of most protein coding genes. Mutations or altered expression of many Mediator subunits have been associated with various medical conditions ranging from cancer to neuropsychiatric illnesses. Mediator complex subunit 12 (MED12), a 45 exon gene on Xq13.1, encodes a subunit of the Mediator's kinase module. MED12 was first implicated in human tumorigenesis when highly specific exon 2 mutations were identified in as many as $70 \%$ of uterine leiomyomas [7]. Functional analyses indicated that these mutations disrupt the interaction between MED12 and Cyclin C-CDK8 and lead to a diminished Mediator-associated CDK kinase activity [8]. Recently, we identified mutations also in exon 1 and showed by expression profiling and functional analyses that they lead to similar unique global gene expression pattern and shared tumorigenic mechanisms as exon 2 mutations [8,9].

Following the observation of specific MED12 mutations in myomas, similar mutations have been searched for in several tumor types [e.g. 10-14]. Mutations have been detected at significant frequency in breast fibroadenomas (59\%) and uterine leiomyosarcomas $(7-20 \%)$, and rarely in extrauterine leiomyomas and leiomyosarcomas, endometrial polyps, and colorectal cancer. To analyze this more thoroughly, we performed a systematic database search utilizing the Catalogue of Somatic Mutations in Cancer (COSMIC) [15]. Interestingly, the search revealed five MED12 mutations in CLL. All these mutations were in exon 1 or 2: three affected the mutation hotspots observed in uterine leiomyomas (codons L36 and G44), the fourth was a missense change altering the last amino acid of exon 1 (E33K), and the fifth resulted in A59P substitution. These data have been retrieved from various recent exome and whole genome sequencing studies, and no mutations in other parts of the gene have been identified. This surprising database finding prompted us to systematically study the contribution of MED12 in CLL.

\section{RESULTS}

Altogether 746 samples from three independent sample series were included in the study. MED12 exons 1 and 2 were successfully sequenced in 709 samples (709/746, 95\%). This analysis revealed mutations in 37 samples (37/709; 5.2\% Supplementary Table 2; two samples had two mutations). Mutations were recurrently identified in each sample series with frequencies of 7.7\% (20/260; CRC samples), 3.6\% (10/274; Sample Bank samples), and $4.0 \%$ (7/175; Finnish samples). There is no obvious bias in the collection or characteristics of the sample series, which could explain the slightly higher mutation frequency in the CRC series.

All the observed mutations were either missense mutations affecting highly conserved amino acids or small insertions or deletions resulting in an in-frame transcript (Figure 1). The most commonly mutated sites were codons G44 (11/39; 28\%, including 9 missense mutations and 2 deletions) and E33 (9/39; 23\%, all missense mutations). Many of the remaining mutations affected the previously reported mutation hotspots (c.100-8T, L36, and Q43). Two variants with unknown significance, a substitution c.1-28G $>\mathrm{T}$ in the UTR and a silent p.R16R in exon 1, were also observed. As their effect on protein function is unclear, they were excluded from the mutation frequency calculations and the association analyses. Detailed information on the observed mutations and the mutation positive samples are presented in Supplementary Table 2.

Association analyses were performed based on the molecular and clinical data available from the patients in the CRC series. Interestingly, MED12 mutations were associated with markers of poor prognosis: unmutated IGHV $[2,3]\left(p=6.8 \times 10^{-4}\right)$, ZAP-70 protein positivity $[16](\geq 20 \%$ expression) $\left(p=7.1 \times 10^{-4}\right)$, and unmethylated $(<20 \%)$ ZAP-70 [17] $\left(p=5.5 \times 10^{-4}\right)$ (Table 1). More specifically, all 20 patients with the MED12 mutation had the established poor prognostic unmutated IGHV disease status, and all 19 who were measured for similar adverse prognostic ZAP-70 methylation were unmethylated. No differences in time to treatment or overall survival were, however, detected.

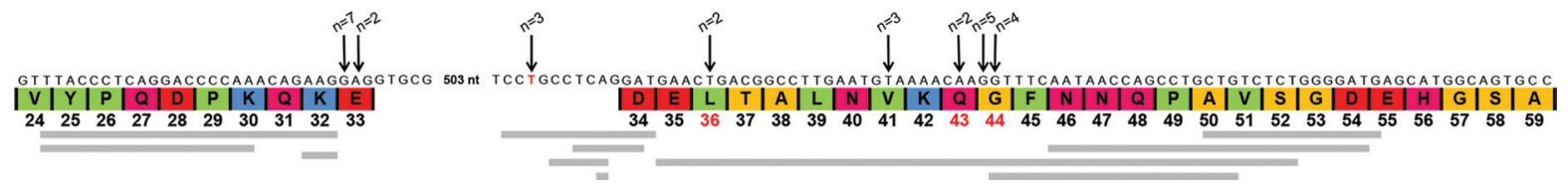

Figure 1: Schematic presentation of MED12 exon 1 and exon 2 mutations in CLLidentified in this study. Amino acids and codon numbers are shown below the DNA sequence. Arrows indicate substitutions and grey bars insertion/deletion mutations, respectively. Numbers above arrows indicate how many times each mutation was detected. Mutation hotspot codons observed in uterine leiomyomas are marked with red. Amino acids are color-coded according to their side-chain $\mathrm{pK}_{\mathrm{a}} \mathrm{s}$ and charge at physiological $\mathrm{pH} 7.4$. Red $=$ negatively charged; green $=$ hydrophobic; yellow $=$ small non-polar, magenta $=$ polar, blue $=$ positively charged . 
Table 1: Demographic and molecular features by MED12 mutation status in CLL Research Consortium sample series. MED12 mutations are associated with markers of poor prognosis in CLL: unmutated IGHV, ZAP-70 protein positivity, and unmethylated ZAP-70.

\begin{tabular}{|c|c|c|c|}
\hline CLL Marker Data & MED12 Not Mutated $(n=240)$ & MED12 Mutated $(n=20)$ & $P$ \\
\hline \multicolumn{4}{|l|}{ Age } \\
\hline Median & 55 & 56 & 0.55 \\
\hline Range & $26-82$ & $44-72$ & \\
\hline \multicolumn{4}{|l|}{ Sex } \\
\hline Male & $169(70 \%)$ & $16(80 \%)$ & 0.45 \\
\hline Female & $71(30 \%)$ & $4(20 \%)$ & \\
\hline \multicolumn{4}{|l|}{ IGHV } \\
\hline Mutated & $79(33 \%)$ & $0(0 \%)$ & $<0.001$ \\
\hline Unmutated $(\geq 98 \% *)$ & $161(67 \%)$ & $20(100 \%)$ & \\
\hline \multicolumn{4}{|l|}{ CD38 } \\
\hline Negative & $115(48 \%)$ & $5(25 \%)$ & 0.06 \\
\hline Positive ( $\geq 20 \%)$ & $125(52 \%)$ & $15(75 \%)$ & \\
\hline \multicolumn{4}{|l|}{ ZAP-70 } \\
\hline Negative & $117(49 \%)$ & $2(10 \%)$ & $<0.001$ \\
\hline Positive $(\geq 20 \%)$ & $123(51 \%)$ & $18(90 \%)$ & \\
\hline \multicolumn{4}{|l|}{ ZAP-70 Methylation } \\
\hline Methylated $(\geq 20 \%)$ & $84(35 \%)$ & $0(0 \%)$ & $<0.001$ \\
\hline UnMethylated $(<20 \%)$ & $153(65 \%)$ & $19(100 \%)$ & \\
\hline Unknown & 3 & 1 & \\
\hline
\end{tabular}

"Sequences showing $98 \%$ or greater homology to the corresponding germ-line IGHV sequence were considered unmutated.

\section{DISCUSSION}

Highly specific MED12 mutations were first observed in benign uterine leiomyomas [7]. Subsequent mutation analyses have revealed recurrent mutations also in breast fibroadenomas and uterine leiomyosarcomas [11-14]. Here, prompted by the systematic analysis of somatic mutation database, we have collected three independent sample series totaling a comprehensive series of more than 700 CLL samples. Mutation screening revealed recurrent mutations in all three series with a combined mutation frequency of 5.2\% (37/709). These results indicate that in addition to solid hormone dependent female tumors, MED12 mutations are involved also in CLL pathogenesis. Significant mutation frequency also implies that MED12 should be recognized as an important cancer gene in CLL.

As in uterine leiomyomas, leiomyosarcomas, and breast fibroadenomas, all MED12 mutations in CLL are missense changes or small in-frame insertions and deletions. The most commonly affected mutation hotspot in all tumor types is codon G44. Despite these similarities in mutation profiles, also an interesting difference was observed. A novel MED12 mutation hotspot was identified in CLL as mutations affecting the last amino acid of exon 1, E33, were seen nine times (Figure 1 and Supplementary Table 2). Similar mutations have previously been observed twice in CLL [18], once in bladder carcinoma [15], and twice in colorectal cancer $[15,19]$. No missense mutations at E33 have been reported in uterine leiomyomas, although a few deletions removing the site have been detected [9]. Altogether 12 of the 39 mutations observed in this study were in exon 1 (31\%) and $27(69 \%)$ in exon 2. The location of mutations in CLL (current study and COSMIC database) is statistically different from the location of mutations in uterine leiomyomas, where nearly all reported mutations $(173 / 178 ; 97 \%)$ have been in exon $2\left(p=6.0 \times 10^{-7}\right)[7$, $9,20]$. Our recent data shows that the Cyclin C-CDK8 binding domain of MED12 consists of its $100 \mathrm{~N}$-terminal amino acids, largely encoded by exons 1 and 2 [8]. Gene expression profiling and functional analyses indicate that mutations within these exons lead to similar expression 
profiles and shared tumorigenic mechanism, involving diminished Mediator-associated kinase activity [8, 9]. Whether the observation of different exon 1 and 2 mutation ratios reflects true tissue specific functional relevance will need to be determined with further research.

Extensive molecular and clinical data were available from one US sample series. Interestingly, association analyses showed significant association between MED12 mutations and unmutated IGHV status, ZAP70 expression, and unmethylated ZAP70, which are wellknown poor prognosis markers in CLL [2, 3, 16, 17]. Possible associations with time to treatment and overall survival were also analyzed, but no differences were observed. The role of MED12 mutational status as a putative prognostic factor will need to be studied further, where our data support investigations in a larger series of high-risk patients adequately powered to detect moderate differences in clinical outcome.

A few MED12 mutations in CLL have been identified in recent large-scale sequencing studies [18, 21-24]. As these have been rare mutation positive cases in independent studies, the significant MED12 mutation frequency in CLL has not been recognized prior to this study. Also our literature search of PubMed with the words "MED12" and "leukemia" did not produce any results. This highlights the utility of comprehensive mutation databases, which facilitate identification of true cancer genes which are mutated recurrently but at moderate frequency.

Our results recognize CLL as the first extrauterine cancer type where the 5 ' terminus of $M E D 12$ is mutated at significant frequency. We also show that these mutations are associated with poor prognosis markers in CLL. Identification of such specific mutations in highly different tumor types -benign uterine leiomyomas and breast fibroadenomas, and a malignant chronic leukemia- is both puzzling and intriguing. While the immediate effects of the mutations on the interactions and kinase activity of the Mediator complex may be similar, the downstream effects on transcription may differ in various tissue types. Overall, the results from this study suggest that MED12 plays a role in the pathogenesis of CLL. Further investigations of $M E D 12$ 's function in CLL are warranted.

\section{METHODS}

Three independent sample series from the United States and Finland comprising altogether 746 samples were collected for the study. The first US series consisted of 278 samples obtained from the CLL Research Consortium (CRC) and the second included 292 samples from The Ohio State University's Human Genetics Sample Bank. The Finnish CLL series, consisting of 176 samples, was obtained from the Helsinki University Central Hospital clinical sample collection. Samples were collected either after signed informed consent (living patients) or with authorization from the Ethics Committee of the Helsinki
University Central Hospital (deceased Finnish patients). The study was approved by the Institutional Review Board of each participating CRC site, The Ohio State University, and the Ethics Review Board of the Hospital District of Helsinki and Uusimaa, Helsinki, Finland.

All MED12 mutations observed in exome or whole genome sequencing studies on CLL have resided in exons 1 and 2. Even though some mutations in other exons might be detectable by targeted sequencing with higher depth of coverage, results from these studies suggest that similarly to uterine leiomyomas, MED12 mutations in CLL are restricted to exons 1 and 2. In this study, mutation screening of exons 1 and 2 was done by direct sequencing using previously described primers $[9,12]$. Samples were sequenced with Applied Biosystems 3730 DNA Analyzer (Life Technologies, Thermo Fischer Scientific Waltham, MA, USA) and analyzed manually and with Mutation Surveyor (Softgenetics, State College, PA, USA) or Lasergene SeqMan Pro (DNASTAR, Madison, WI, USA) softwares. See Supplemental Data for more details on the sample series and methods.

Molecular and clinical data were available from patients in the CRC series. Associations between the MED12 mutations and various demographic and molecular characteristics were analyzed using Fisher's exact and Wilcoxon rank sum tests for categorical and continuous variables, respectively.

\section{ACKNOWLEDGMENTS}

The authors would like to thank the patients for participating in this study. Ilene Comeras is thanked for procuring CLL patient samples (The Ohio State University's Human Genetics Sample Bank), and Melanie Davis, Lori Nelsen, Christopher Bigley, Sari Nikkola, Eeva Lind, Anne Gesterberg, Markus Koljonen, Saara Vaalas, Henna Ikäheimo, and Sirpa Soisalo for technical assistance. This study was supported by the Academy of Finland (Academy Research Fellow grants 260370 and 265124 for PV), the Sigrid Jusélius Foundation, the Cancer Society of Finland, the Emil Aaltonen Foundation, the K. Albin Johansson Foundation (for KK), the Leukemia SPORE (grant P50-CA140158 for JCB and AdlC), NIH grant P30 CA16058 to the OSU Comprehensive Cancer Center, Leukemia and Lymphoma Society, D Warren Brown Family Foundation (for JCB), the Jane and Aatos Erkko Foundation, the Jenny and Antti Wihuri Foundation / the Foundation's Post Doc Pool, the Paulo Foundation, and the Maud Kuistila Memorial Foundation (for TMJ).

\section{REFERENCES}

1. Zhang S, Kipps TJ. The pathogenesis of chronic lymphocytic leukemia. Annu Rev Pathol. 2014; 9:103-118.

2. Damle RN, Wasil T, Fais F, Ghiotto F, Valetto A, Allen SL, Buchbinder A, Budman D, Dittmar K, Kolitz J, Lichtman 
SM, Schulman P, Vinciguerra VP, et al. Ig V gene mutation status and CD38 expression as novel prognostic indicators in chronic lymphocytic leukemia. Blood. 1999; 94:1840-1847.

3. Hamblin TJ, Davis Z, Gardiner A, Oscier DG, Stevenson FK. Unmutated ig $\mathrm{V}(\mathrm{H})$ genes are associated with a more aggressive form of chronic lymphocytic leukemia. Blood. 1999; 94:1848-1854.

4. Byrd JC, Rai K, Peterson BL, Appelbaum FR, Morrison VA, Kolitz JE, Shepherd L, Hines JD, Schiffer CA, Larson RA. Addition of rituximab to fludarabine may prolong progression-free survival and overall survival in patients with previously untreated chronic lymphocytic leukemia: An updated retrospective comparative analysis of CALGB 9712 and CALGB 9011. Blood. 2005; 105:49-53.

5. Vogelstein B, Papadopoulos N, Velculescu VE, Zhou S, Diaz LA Jr, Kinzler KW. Cancer genome landscapes. Science. 2013; 339:1546-1558.

6. Martinez-Trillos A, Quesada V, Villamor N, Puente XS, Lopez-Otin C, Campo E. Recurrent gene mutations in CLL. Adv Exp Med Biol. 2013; 792:87-107.

7. Mäkinen N, Mehine M, Tolvanen J, Kaasinen E, Li Y, Lehtonen HJ, Gentile M, Yan J, Enge M, Taipale M, Aavikko M, Katainen R, Virolainen E, et al. MED12, the mediator complex subunit 12 gene, is mutated at high frequency in uterine leiomyomas. Science. 2011; 334:252-255.

8. Turunen M, Spaeth JM, Keskitalo S, Park MJ, Kivioja T, Clark AD, Mäkinen N, Gao F, Palin K, Nurkkala H, Vähärautio A, Aavikko M, Kämpjärvi K, et al. Uterine leiomyoma-linked MED12 mutations disrupt mediatorassociated CDK activity. Cell Rep. 2014; 7:654-660.

9. Kämpjärvi K, Park MJ, Mehine M, Kim NH, Clark AD, Bützow R, Böhling T, Böhm J, Mecklin JP, Järvinen H, Tomlinson IP, van der Spuy ZM, Sjöberg J, et al. Mutations in exon 1 highlight the role of MED12 in uterine leiomyomas. Hum Mutat. 2014; 35:1136-1141.

10. Je EM, Kim MR, Min KO, Yoo NJ, Lee SH. Mutational analysis of MED12 exon 2 in uterine leiomyoma and other common tumors. Int J Cancer. 2012; E1044-E1047.

11. Perot G, Croce S, Ribeiro A, Lagarde P, Velasco V, Neuville A, Coindre JM, Stoeckle E, Floquet A, Macgrogan G, Chibon F. MED12 alterations in both human benign and malignant uterine soft tissue tumors. PLoS One. 2012; 7:e40015.

12. Kämpjärvi K, Mäkinen N, Kilpivaara O, Arola J, Heinonen HR, Böhm J, Abdel-Wahab O, Lehtonen HJ, Pelttari LM, Mehine M, Schrewe H, Nevanlinna H, Levine RL, et al. Somatic MED12 mutations in uterine leiomyosarcoma and colorectal cancer. Br J Cancer. 2012; 107:1761-1765.

13. Ravegnini G, Marino-Enriquez A, Slater J, Eilers G, Wang Y, Zhu M, Nucci MR, George S, Angelini S, Raut CP, Fletcher JA. MED12 mutations in leiomyosarcoma and extrauterine leiomyoma. Mod Pathol. 2012; 26:743-749.

14. Lim WK, Ong CK, Tan J, Thike AA, Ng CC, Rajasegaran V, Myint SS, Nagarajan S, Nasir ND, McPherson JR, Cutcutache I, Poore G, Tay ST, et al. Exome sequencing identifies highly recurrent MED12 somatic mutations in breast fibroadenoma. Nat Genet. 2014; 46:877-880.

15. Forbes SA, Bhamra G, Bamford S, Dawson E, Kok C, Clements J, Menzies A, Teague JW, Futreal PA, Stratton MR. The catalogue of somatic mutations in cancer (COSMIC). Curr Protoc Hum Genet. 2008; Chapter 10: Unit 10.11 .

16. Wiestner A, Rosenwald A, Barry TS, Wright G, Davis RE, Henrickson SE, Zhao H, Ibbotson RE, Orchard JA, Davis Z, Stetler-Stevenson M, Raffeld M, Arthur DC, et al. ZAP-70 expression identifies a chronic lymphocytic leukemia subtype with unmutated immunoglobulin genes, inferior clinical outcome, and distinct gene expression profile. Blood. 2003; 101:4944-4951.

17. Claus R, Lucas DM, Stilgenbauer S, Ruppert AS, Yu L, Zucknick M, Mertens D, Buhler A, Oakes CC, Larson RA, Kay NE, Jelinek DF, Kipps TJ, et al. Quantitative DNA methylation analysis identifies a single $\mathrm{CpG}$ dinucleotide important for ZAP-70 expression and predictive of prognosis in chronic lymphocytic leukemia. J Clin Oncol. 2012; 30:2483-2491.

18. Wang L, Lawrence MS, Wan Y, Stojanov P, Sougnez C, Stevenson K, Werner L, Sivachenko A, DeLuca DS, Zhang L, Zhang W, Vartanov AR, Fernandes SM, et al. SF3B1 and other novel cancer genes in chronic lymphocytic leukemia. N Engl J Med. 2011; 365:2497-2506.

19. Cancer Genome Atlas Network: Comprehensive molecular characterization of human colon and rectal cancer. Nature. 2012; 487:330-337.

20. Mäkinen N, Heinonen HR, Moore S, Tomlinson IP, van der Spuy ZM, Aaltonen LA. MED12 exon 2 mutations are common in uterine leiomyomas from south african patients. Oncotarget. 2011; 2:966-969.

21. Quesada V, Conde L, Villamor N, Ordonez GR, Jares P, Bassaganyas L, Ramsay AJ, Bea S, Pinyol M, MartinezTrillos A, Lopez-Guerra M, Colomer D, Navarro A, et al. Exome sequencing identifies recurrent mutations of the splicing factor SF3B1 gene in chronic lymphocytic leukemia. Nat Genet. 2011; 44:47-52.

22. Landau DA, Carter SL, Stojanov P, McKenna A, Stevenson K, Lawrence MS, Sougnez C, Stewart C, Sivachenko A, Wang L, Wan Y, Zhang W, Shukla SA, et al. Evolution and impact of subclonal mutations in chronic lymphocytic leukemia. Cell. 2013; 152:714-726.

23. Schuh A, Becq J, Humphray S, Alexa A, Burns A, Clifford R, Feller SM, Grocock R, Henderson S, Khrebtukova I, Kingsbury Z, Luo S, McBride D, et al. Monitoring chronic lymphocytic leukemia progression by whole genome sequencing reveals heterogeneous clonal evolution patterns. Blood. 2012; 120:4191-4196.

24. Damm F, Mylonas E, Cosson A, Yoshida K, Della Valle V, Mouly E, Diop M, Scourzic L, Shiraishi Y, Chiba K, Tanaka H, Miyano S, Kikushige Y, et al. Acquired initiating mutations in early hematopoietic cells of CLL patients. Cancer Discov. 2014; 4:1088-1101. 